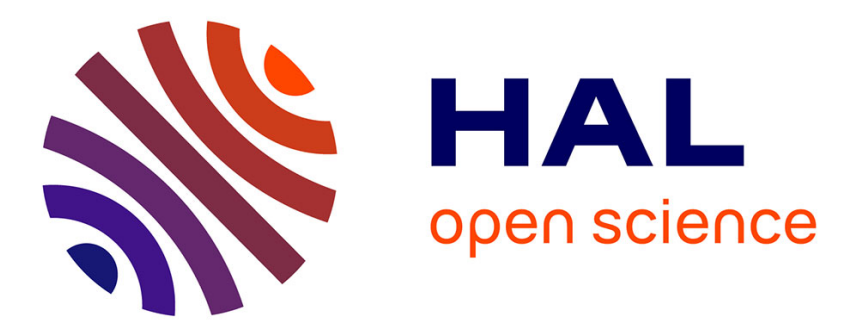

\title{
Enhanced optoelastic interaction range in liquid crystals with negative dielectric anisotropy
}

Francesco Simoni, S. Lalli, Liana Lucchetti, L. Criante, Etienne Brasselet

\section{To cite this version:}

Francesco Simoni, S. Lalli, Liana Lucchetti, L. Criante, Etienne Brasselet. Enhanced optoelastic interaction range in liquid crystals with negative dielectric anisotropy. Applied Physics Letters, 2014, 104 (1), pp.011112 (1-4). 10.1063/1.4861473 . hal-00927719

\section{HAL Id: hal-00927719 \\ https://hal.science/hal-00927719}

Submitted on 23 Mar 2015

HAL is a multi-disciplinary open access archive for the deposit and dissemination of scientific research documents, whether they are published or not. The documents may come from teaching and research institutions in France or abroad, or from public or private research centers.
L'archive ouverte pluridisciplinaire HAL, est destinée au dépôt et à la diffusion de documents scientifiques de niveau recherche, publiés ou non, émanant des établissements d'enseignement et de recherche français ou étrangers, des laboratoires publics ou privés.

\section{(c) (1) $\$$}

Distributed under a Creative Commons Attribution - NonCommerciall 4.0 International 


\title{
Enhanced optoelastic interaction range in liquid crystals with negative dielectric anisotropy
}

\author{
F. Simoni, ${ }^{1}$ S. Lalli, ${ }^{1}$ L. Lucchetti, ${ }^{1}$ L. Criante,${ }^{2}$ and E. Brasselet ${ }^{3}$ \\ ${ }^{1}$ Dipartimento di Scienze e Ingegneria della Materia, dell' Ambiente ed Urbanistica and CNISM, Università \\ Politecnica delle Marche, Ancona, Italy \\ ${ }^{2}$ Center for Nano Science and Technology@PoliMi, Istituto Italiano di Tecnologia, Via Giovanni Pascoli, \\ 70/3, 20133 Milano, Italy \\ ${ }^{3}$ Univ. Bordeaux and CNRS, Laboratoire Ondes et Matière d'Aquitaine, UMR 5798, F 33400 Talence, France
}

\begin{abstract}
We demonstrate that the long-range interaction between surface-functionalized microparticles immersed a nematic liquid crystal a "nematic colloid" and a laser-induced "ghost colloid" can be enhanced by a low-voltage quasistatic electric field when the nematic mesophase has a negative dielectric anisotropy. The optoelastic trapping distance is shown to be enhanced by a factor up to 2.5 in presence of an electric field. Experimental $\mathrm{d}$ ata a re q uantitatively $\mathrm{d}$ escribed $\mathrm{w}$ ith a theoretical model accounting for the spatial overlap between the orientational distortions around the microparticle and those induced by the trapping light beam itself.
\end{abstract}

During the last decade, great efforts have been made to propose efficient strategies that enable self-assembling of microparticles, with the aim at fabricating tailored-made microscopic architectures in two and three dimensions. The intrinsic long-range orientational order of liquid crystals mesophases and their ability to exhibit topological defects have been recognized as the key features that make liquid crystals suitable for this task. In particular, liquid crystals have been recognized as useful active host media enabling the building up of crystalline assemblies of microparticles ${ }^{1-3}$ and also as three-dimensional templates. ${ }^{4}$ In addition, since liquid crystals are highly sensitive to external fields (for instance temperature, electric, magnetic, or electromagnetic fields) assembling options that rely on them are naturally endowed with tunable and reconfigurable properties.

In general, the developed approaches that involve liquid crystals are based on the use of spherical micron-sized particles dispersed in nematic liquid crystals films and often take advantage of optical tweezing techniques. Indeed, focused laser beams enable easy contactless trapping and three-dimensional spatial displacement of microscopic objects towards ordered structures. A number of important observations have been reported ${ }^{5}$ and, among them, the unexpected optical trapping of microparticles having a refractive index lower than the one of the surrounding liquid crystalline medium. ${ }^{6}$ It has been pointed out that the origin of the force experienced by such a "nematic colloid," which attracts it towards the focal spot where local optical reorientation of the liquid crystal acts as a "ghost colloid," differs from the one arising from light intensity gradients as is usually the case for optical tweezers operating with high numerical aperture (NA). In fact, it has been shown that a light field obtained from a microscope objective with sufficiently modest NA value, which prevents from any possible conventional trapping based on optical gradient forces, can actually trap a nematic colloid, see Ref. 7 where NA $=0.45$. To distinguish such an effect from the conventional trapping phenomenon, we refer to it as optoelastic trapping.
In contrast to conventional trapping that is based on the minimization of the electromagnetic energy in the considered medium, optoelastic trapping basically results from the minimization of the elastic energy of the system, thereby being a special feature of soft condensed matter systems. Importantly, the optoelastic effect can originate either from (i) the long-range orientational interaction between the nematic and the ghost colloids or (ii) the gradient of the order parameter (which preserves the orientational configuration below the nematic-isotropic clearing point) induced by local temperature rise at the focal spot as a result of partial absorption of the light field. Experimentally, the latter effect has been recently reported ${ }^{8}$ using conventional optical tweezers and focusing the light beam on a thin nematic film (5 $7 \mu \mathrm{m}$ ) sandwiched between two glass substrates provided with ITO (Indium Tin Oxide) coating.

An attractive feature of the case (i) is that the optoelastic manipulation of a microparticle can be electrically tuned since optical reorientation of the director field (i.e., the local average orientation of the liquid crystal molecules) is involved. This was shown in a previous study where we demonstrate, both experimentally and theoretically, that the long-range optoelastic interaction can be electrically screened in the case of a nematic with positive dielectric anisotropy. ${ }^{9}$ In Ref. 9, the proposed model neglects heating effects, takes into account the electrically induced reorientation along the electric field direction and includes the elastic interaction between the nematic and ghost colloids that arise from the spatial overlap between the orientational distortions around the microparticle and those induced by the trapping light beam itself.

In contrast, in the case of liquid crystals with negative dielectric anisotropy, the latter model predicts an optoelastic interaction range $\Lambda$ (i.e., the distance above which the nematic and ghost colloids do not longer interact) of the form ${ }^{9}$

$$
\Lambda=\frac{d_{\text {cutoff }}}{\sqrt{ } 1-\tilde{V}^{2}},
$$


where $d_{\text {cutoff }}$ is the interparticle distance above which the real and ghost colloids no longer interact at zero applied voltage $V$ and $\tilde{V}=V / V_{F}$ with $V_{F}$ the Fréedericksz transition voltage. It appears from Eq. (1) that $\Lambda$ diverges as $V \rightarrow V_{F}$, when the whole nematic film is at the onset of the electrically induced orientational instability threshold.

The principle of electrically enhanced optoelastic interaction range is sketched in Fig. 1. Such a situation is interesting from an applicative point of view since it formally paves the way towards large-distance manipulation of colloidal particles. Noteworthy, large-area optoelastic manipulation of colloidal particles in liquid crystals has been demonstrated recently, however using photoresponsive molecular surface monolayers. ${ }^{10}$ Here, we bring a quantitative experimental evidence of the earlier prediction given by Eq. (1) by using "negative" pure nematic liquid crystals.

Experiments are performed using homemade $50 \mu \mathrm{m}$ thick nematic liquid crystal film sandwiched between two ITO coated glass substrates. The nematic compound is ZLI-4788 (from Merck) with refractive index parallel the director $n_{\|}=1.6567$, optical birefringence $\Delta n=0.1647$ at $\lambda=589 \mathrm{~nm}$, dielectric anisotropy $\Delta \varepsilon=\varepsilon_{\|}-\varepsilon_{\perp}=-5.7$ at $1 \mathrm{kHz}$ frequency and bend Frank elastic constant $K_{3}=18.9 \mathrm{pN}$. Both substrates were treated by dimethyloctadecyl[3-(trimethoxysilyl) propyl] ammonium chloride (DMOAP, from FLUKA) in order to promote strong homeotropic anchoring (the director is perpendicular to the substrates at both ends of the film). Nematic colloids are obtained from silica spheres (from Bang Laboratories Inc.) with $2.5 \mu \mathrm{m}$ radius and refractive index 1.37 , also covered by DMOAP, which were dispersed in the nematic before capillary filling of the sample. The light-induced ghost colloid is driven by a linearly polarized Gaussian laser beam at $532 \mathrm{~nm}$ wavelength focused at normal incidence onto the sample.
The question whether the situation (i) may occur independently of (ii) is a practical issue worth to discuss since Eq. (1) is based on a model that discards any thermal effects. In fact, the effective NA of our focusing system (microscope objective with $\mathrm{NA}=0.25$ used in underfilling conditions) is about 0.08 , with an actual intensity on the sample that is typically 10100 times lower than the minimum one used in experiments performed under conventional trapping conditions. $^{6,11}$ Moreover, the laser beam of total incident power $10 \mathrm{~mW}$ is focused in the center of the cell in order to minimize heating effects due to ITO absorption. In addition, crossed polarizers imaging of the trapping area gives a bright spot, which indicates optically induced anisotropy. Finally, we notice the observation of an anisotropic attractive potential. Indeed, at fixed pump beam polarization, different positions of the particle lead generally to curved trajectories except for the one that corresponds to "easy-axis" optoelastic trapping, for which the nematic colloid trajectory is a straight line $^{11}$ as is the case in the experiments reported here. All this supports the fact that the observed laser induced distorted director region results from optical reordering rather than heating phenomena associated with isotropic attraction potential. $^{8}$

Measurements are performed on an isolated dipolar colloidal particle by following its real-time dynamics by CCD camera video imaging. This is done by using a white light illumination from the opposite side to that of the trapping laser beam. The experiment basically consists to compare the dynamics of various nematic colloid trajectories as a function of the applied voltage $V$ at fixed initial interparticle distance $d_{0}$. This is illustrated in Fig. 2 for $d_{0}\left(d_{\text {cutoff }}=23 \mu \mathrm{m}\right.$ (where $d_{\text {cutoff }} \sim 23 \mu \mathrm{m}$ is determined following a protocol described in Ref. 9) and $V=0,0.5$, and 1.5 V. From this figure, it appears that the larger is the applied voltage, the smaller is the optoelastic trapping time. This qualitatively

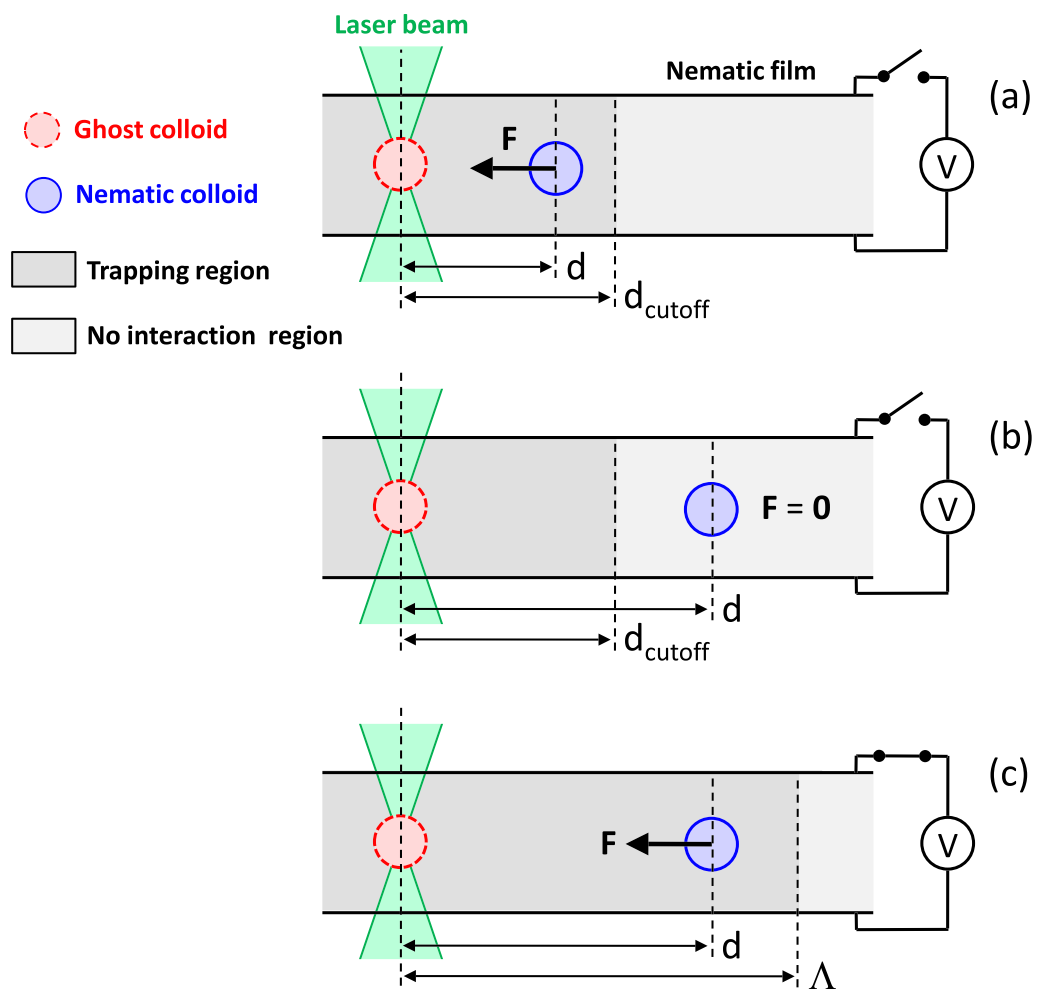

FIG. 1. Schematic of the electrically induced optoelas tic interaction range between a laser induced "ghost colloid" and a "nematic colloid" made of a real micro particle embedded in nematic liquid crystal with nega tive dielectric anisotropy. Without applied voltage, the interaction is screened above the cutoff distance $d_{\text {cutoff }}$ ( $a$ and b) whereas optoelastic trapping is at work up to the interaction range $\Lambda>d_{\text {cutoff }}$ when $V / 0$ (c). 


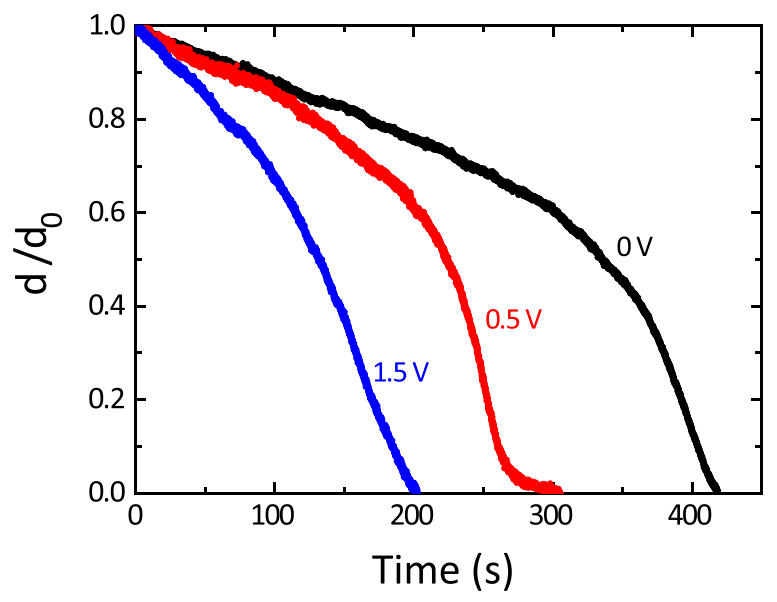

FIG. 2. Reduced interparticle distance $d / d_{0}$ for an initial distance $d_{0} \quad 23 \mu \mathrm{m}$ between nematic and ghost colloids as a function of time for different values of the applied voltage $V$.

demonstrates that the optoelastic interaction range is increased. Indeed, the electric field, which tends to realign the director in the plane of the film, increases the overlap between the reoriented area associated with the nematic and ghost colloids. Since the attraction potential has been shown to directly depend on such an overlap, ${ }^{7,9}$ this means that the mutual attraction potential is enhanced.

More quantitatively, the enhancement of the attraction potential can be retrieved by considering, the following expression for the attractive force $F$ exerted on the nematic colloid, $F=-A / r^{2}$, as previously demonstrated in Ref. 7. In fact, since one can safely neglect the inertial term in the equation of motion, the balance of forces between the optoelastic force and the viscous force, which we assume to be described by the Stokes formula for a sphere, leads to the expression $F=6 \pi R \eta_{\text {eff }}(\mathrm{d} d / \mathrm{d} t)$, with $R$ the silica sphere radius and $\eta_{\text {eff }}$ the effective dynamic viscosity of the medium in our configuration (homeotropic anchoring, dipolar colloid oriented in a direction perpendicular to the that of its motion). Due to the lack of information on $\eta_{\text {eff }}$ for our nematic compound, an estimate is made noting that theoretically established value of $\eta_{\text {eff }}$ for the nematic MBBA and $5 \mathrm{CB}$ roughly satisfy $\eta_{\mathrm{eff}} \sim\left(\eta_{1}+\eta_{3}\right) / 2$ with $\eta_{1,3}$ the first and third Miesowicz viscosities. ${ }^{12}$ By doing so, from the measured values of $\eta_{1,3}$ for the nematic ZLI-4788, ${ }^{13}$ we get $\eta_{\text {eff }}$ $\sim 200$ mPas. As detailed in Ref. 9, it is therefore possible to evaluate the coefficient $A$ by fitting $F(d)$, which is typically done over the first ten of microns of the particle displacement. The results are presented in Fig. 3, which corresponds to the data shown in Fig. 1. We thus have quantitatively demonstrated that the larger is the applied voltage, the larger is the interaction potential. One may argue that a direct measurement of the effective viscosity could have been obtained from the study of the Brownian motion of the nematic colloid, which would a priori lead to a refined estimation of A. However, let us recall that the way $\mathrm{F}$ is expressed as a function of $\mathrm{A}$ is somewhat empirical in view of the complexity of the actual interplay between light, electric field and the medium, which in fine limits the impact of any refined quantitative measurement of the dissipation coefficient. Finally, it is worth to mention that this would obviously not alter our

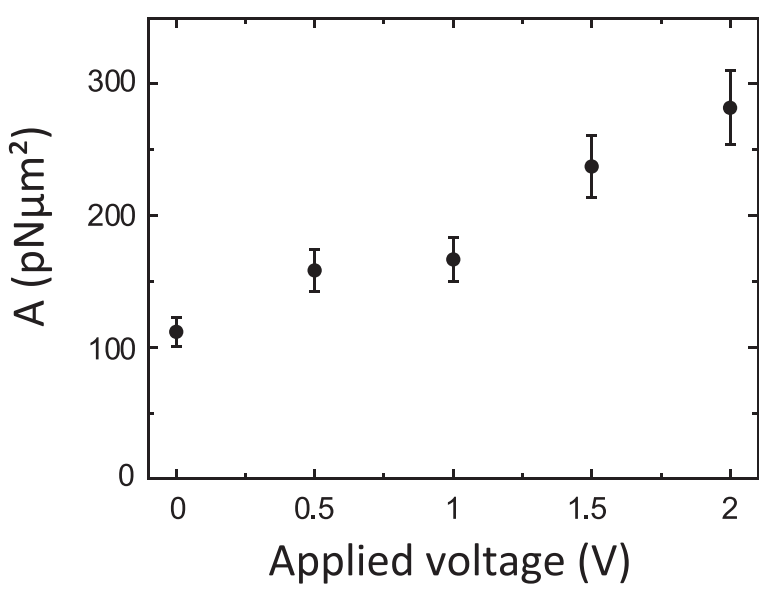

FIG. 3. Electrically enhanced attractive optoelastic interaction potential: parameter $A$ as a function of the applied voltage for the dynamics of the distance $d(t)$ between the nematic and ghost colloids presented in Fig. 2.

conclusions regarding the enhancement of the optoelastic trapping interaction range.

In a second step, we have experimentally measured the optoelastic interaction range $\Lambda$ by itself. Practically, $\Lambda$ is determined as the minimum distance between the nematic and ghost colloids for which $F=0$. The experiment first consists in the determination of $d_{\text {cutoff }}$, which is performed at zero applied voltage. The initial interparticle distance $d_{0}$ is fixed and the laser is switched on. The nematic colloid is then imaged during $30 \mathrm{~min}$. If motion is detected, the experiment is restarted but with a larger initial distance $d_{0}$ until no motion is detected, which defines the optoelastic interaction range at zero voltage, $d_{\text {cutoff }}$. Then, the system is prepared with $d_{0}>d_{\text {cutoff }}$, for which no interaction occurs at $V=0$ (see Fig. 1(b)), and the applied voltage is progressively increased until motion is eventually detected within a $30 \mathrm{~min}$ observation time. Following this procedure, the chosen $d_{0}$ is a measure of $\Lambda$ at the reached voltage. The results are shown in Fig. 4 where $\Lambda / d_{\text {cutoff }}$ is plotted vs the reduced voltage $V / V_{\mathrm{F}}$.

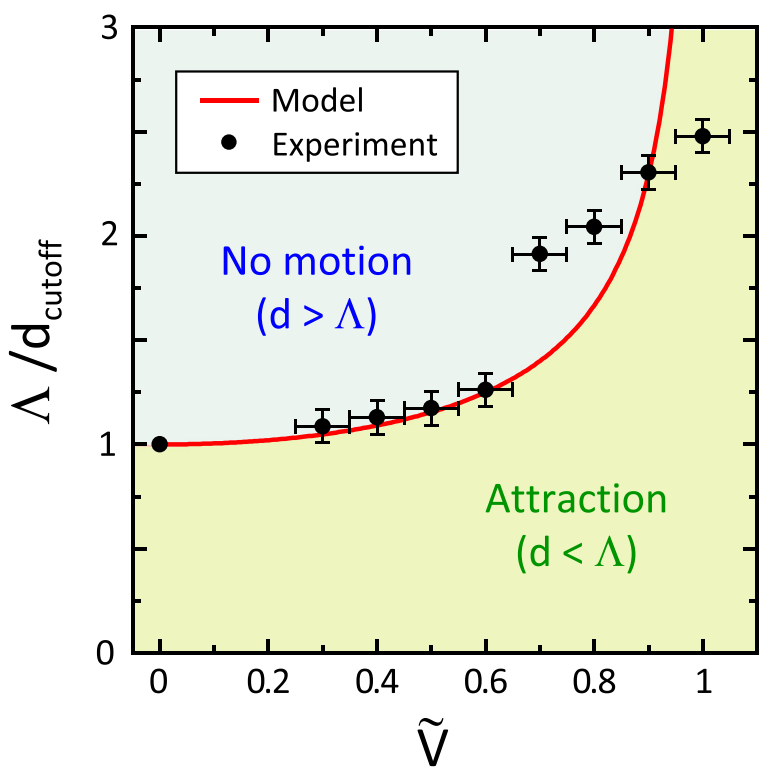

FIG. 4. Reduced interaction range $\Lambda / d_{\text {cutoff }}$ vs reduced voltage $V / V_{\mathrm{F}}$. Markers: experimental data. Curve: best fit from Eq. (1). 
The data are then confronted to the prediction given by Eq. (1) by using $V_{\mathrm{F}}$ as the single fitting parameter, see curve in Fig. 4. Despite a resulting fair agreement, the best fit value $V_{\mathrm{F}}=0.95 \pm 0.03 \mathrm{~V}$, however, significantly departs from the expected value for the electric Fréedericksz threshold by a factor of two. Indeed, $V_{\mathrm{F}}=\pi\left[K_{3} /\left(\varepsilon_{0}|\Delta \varepsilon|\right]^{1 / 2}\right.$, which gives $V_{\mathrm{F}}=1.92 \mathrm{~V}$. A reason might be found in the basic assumption of the model presented in Ref. 9, namely $\left(\mathrm{d}_{\text {cutoff }}\right)^{2} \gg(L / \pi)^{2}$, which is not that much safely satisfied for the nematic used here. Still, in overall, the presented data show an enhancement the optoelastic interaction range by a factor up to 2.5 , even if the divergence of $\Lambda$ is not clearly shown. A possible explanation is the fact that any deviation of the sample from the ideal homeotropic parallel slab of liquid crystal (e.g., due to the presence of the microparticles) is expected to alter the homogeneous character of the electrical Fréedericksz bifurcation, thereby fading the predicted divergence of $\Lambda$ given by Eq. (1). Indeed, the divergence of $\Lambda$ is associated with the divergence of the coherence length as $V$ tends to $V_{\mathrm{F}}$. Therefore, any spatial inhomogeneity is expected to introduce deviation to Eq. (1). Also, from a practical point of view, we note that the present experimental analysis is restricted to a scale of tenths of microns due to the limited field of view of the visualization system, which has restricted our investigations to interparticle distances below $\sim 100 \mu \mathrm{m}$.

Summarizing, it is formally possible to take control over the intrinsic finite optoelastic interaction range of microparticles immersed in liquid crystals and manipulated by light. This has been experimentally demonstrated by using nematic liquid crystals with negative dielectric anisotropy, for which a diverging interaction range has been predicted. Finally, it is worth mentioning that the use of dual frequency liquid crystals, whose sign of the dielectric anisotropy can be controlled by mere change of the applied voltage frequency, would merge in a single medium the capabilities of present work with those already demonstrated in Ref. 9 for positive dielectric anisotropy, i.e., electrically tunable screening of the optoelastic interaction.

${ }^{1}$ P. Poulin, H. Stark, T. C. Lubensky, and D. A. Weitz, Science 275, 1770 (1997).

${ }^{2}$ M. Ravnik, M. Skarabot, S. Zumer, U. Tkalec, I. Poberaj, D. Babic, N. Osterman, and I. Musevic, Phys. Rev. Lett. 99, 247801 (2007).

${ }^{3}$ I. Musevic, M. Skarabot, U. Tkalec, M. Ravnik, and S. Zumer, Science 313, 954 (2006).

${ }^{4}$ M. Ravnik, G. P. Alexander, J. M. Yeomans, and S. Zumer, Proc. Natl. Acad. Sci. U.S.A. 108, 5188 (2011).

${ }^{5}$ R. P. Trivedi, D. Engstrom, and I. I. Smalyukh, J. Opt. 13, 044001 (2011).

${ }^{6}$ I. Musevic, M. Skarabot, D. Babic, N. Osterman, I. Poberaj, V. Nazarenko, and A. Nych, Phys. Rev. Lett. 93, 187801 (2004).

${ }^{7}$ L. Lucchetti, L. Criante, F. Bracalente, F. Aieta, and F. Simoni, Phys. Rev. E 84, 021702 (2011).

${ }^{8}$ M. Skarabot, Z. Lokar, and I. Musevic, Phys. Rev. E 87, 062501 (2013).

${ }^{9}$ L. Criante, F. Bracalente, L. Lucchetti, F. Simoni, and E. Brasselet, Soft Matter 9, 5459 (2013).

${ }^{10}$ A. Martinez, H. C. Mireles, and I. I. Smalyukh, Proc. Natl. Acad. Sci. U.S.A. 108, 20891 (2011).

${ }^{11}$ M. Skarabot, M. Ravnik, D. Babic, N. Osterman, I. Poberaj, S. Zumer, I. Musevic, A. Nych, U. Ognysta, and V. Nazarenko, Phys. Rev. E 73, 021705 (2006).

${ }^{12}$ H. Stark and D. Ventzki, Phys. Rev. E 64, 031711 (2001).

${ }^{13}$ P. J. M. Vanbrabant, J. Beeckman, K. Neyts, R. James, and F. A. Fernandez, Appl. Phys. Lett. 95, 151108 (2009). 